\title{
Diversidad lingüístico-cultural e interculturalismo en la escuela andaluza. Un análisis de políticas educativas
}

\author{
Cultural and linguistic diversity and interculturalism in andalusian school: an análisis of \\ education policies
}

\section{Olmos-Alcaraz, Antonia}

Universidad de Granada

\begin{abstract}
This paper is based on some research projects about immigration and education in Spain where I have been working on. The objectives of this work are: 1). To show the linguistic diversity of the students at Andalusian school, due to the migratory phenomenon; and 2). To analyse the educational policies designed to manage the linguistic diversity in schools. The results -obtained by conducting questionaries, interviews and participant observation- indicate that, due to the increase of linguistic diversity in the last decade, some educational policies have been designed to manage the new linguistic and cultural situation, but also indicate that there are 'different kinds of policies for different kinds of students'. As a conclusion of this paper, I make a critical approach of how the government is treating the arrivals of 'new students', and how the different languages have been treated, because some of them have been more recognized than others.
\end{abstract}

Keywords: Interculturality, Migrations, School, Language, Cultural diversity

\section{Resumen}

El texto muestra parte del trabajo realizado a raíz de la participación en diversas investigaciones sobre inmigración y escuela en España. Los objetivos del artículo son: 1). Mostrar la diversidad lingüística de los estudiantes en la escuela en contexto andaluz, producto de los movimientos migratorios internacionales; y 2). Analizar las políticas educativas diseñadas para gestionar dicha diversidad. Los resultados que se exponen -fruto de la realización de cuestionarios, entrevistas y observación participante- indican que, para responder al incremento de la diversidad, las administraciones públicas han diseñado dispositivos para enseñar español al alumnado extranjero y programas para enseñar lenguas como el inglés, francés o alemán, a los que no asisten normalmente los/as alumnos/as inmigrantes, quedando fuera de estas políticas lingüísticas las "lenguas de los/as inmigrantes". En la discusión se reflexiona

Reception Date 2015August 08

Approval Date 2016 October 23

Publication Date: 2016 October 23

Fecha de recepción 2015 Agosto 08

Fecha de aprobación 2016 Octubre 23

Fecha de publicación 2016 Octubre 23 sobre el trato diferenciador que se está dando con ello al alumnado, de tal forma que podemos hablar de "distintos tipos de políticas para distintos tipos de alumnado".

Palabras clave: Interculturalidad, Migraciones, Escuela, Lengua, Diversidad cultural

Afirmar la homogeneidad de España en términos lingüísticos y culturales siempre ha sido una cuestión polémica, tintada de intereses políticos explícitos. La diversidad lingüística y cultural en España es un hecho histórico. Además en el momento actual, y desde hace un par de décadas, se está asistiendo a un proceso de crecimiento progresivo de dicha diversidad, debido a los movimientos migratorios internacionales que tienen como destino España. Esta "nueva 
diversidad" convive ahora con las heterogeneidades históricas del país. Desde otros contextos se vienen definiendo hace ya algún tiempo estas nuevas situaciones, generadas a la luz de las sociedades globalizadas, con los conceptos de "diversidad de diversidades” (Wallman, 2003) y/o "súperdiversidad" (Vertovec, 2007; Meissner \& Vertovec, 2015). Dichas propuestas teóricas tratan de dar cuenta de la complejización y el funcionamiento de la heterogeneidad actuales más allá de consideraciones ancladas en conceptos de diversidad cultural reduccionistas, que entienden ésta como diversidad étnica esencialmente. Ello es aplicable también en gran medida al contexto estudiado, y al contexto de los países del sur de Europa (Padilla, Azevedo \& OlmosAlcaraz, 2015) en general, en tanto que sociedades interconectadas, glocales, transnacionales, donde las migraciones han hecho que las poblaciones sean tremendamente complejas en su composición: origen, lengua, raza/etnia, edad, género, clase social, estatus migratorio, etc. serían solo algunas de las categorías de la "súperdiversidad".

El ámbito educativo formal es uno de los espacios donde más visibilidad tiene este fenómeno, de tal modo que las distintas administraciones (central y autonómicas) han venido diseñando e implementando desde hace ya algún tiempo toda una serie de políticas educativas para gestionar esta realidad tan diversa. Véanse los trabajos de Antolinez y García-Cano (2011), Jiménez (2012), OlmosAlcaraz (2010), Rubio (2013), Márquez y García-Cano (2014) y Castilla (2014), entre otros, para el caso de Andalucía; Alegre (2011), Carrasco (2011) y Simó, Pámies, Collet-Sabé y Tort (2014), entre otros, para Cataluña; o Poveda, Jociles y Franzé (2009), Sánchez y García (2011), Del Olmo (2012) y Cucalón (2014), entre otros, para conocer sobre la Comunidad de Madrid. Así mismo contamos ya con trabajos que exploran las formas de gestionar la diversidad relacionada con la inmigración a nivel estatal (García, Fernández, Rubio \& Soto, 2011), y que ofrecen análisis comparativos entre las distintas Comunidades Autónomas.

Las características de dichas políticas responden en gran medida a las construcciones socio-culturales que la sociedad española en su conjunto hace sobre el fenómeno migratorio, y sus protagonistas: los/as inmigrantes. Es por ello que distintos autores han manifestado que una consideración problemática de las migraciones lleva aparejada una gestión de las mismas, también, como problema (ZapataBarrero, 2001; Santamaría, 2002; OlmosAlcaraz, 2012; Sebastiani, 2015). En este contexto -en materia de políticas de atención educativa al alumnado de nacionalidad extranjera- vemos cómo desde la década de los años noventa toma fuerza en el país el paradigma de la interculturalidad (Dietz, 2012), reduciendo con ello en gran medida la puesta en práctica de lo que se define como "Educación Intercultural” a la atención/actuación con alumnos que cumplen unas determinadas características (Franzé, 2003; Ortiz, 2005; Olmos-Alcaraz, 2010; García et al., 2011; Carrasco, 2011; Fernández \& García, 2015). Es decir, en el contexto español el interculturalismo empezó a tomar fuerza para dar respuesta a la llegada de población inmigrante extranjera, y no a otras diversidades históricas del país (García \& Martínez, 2002; Carrasco, 2003; Del Olmo \& Hernández, 2004). Se visibilizan en este proceso unas categorías de la diversidad en mayor medida que otras: la lengua y la cultura (entendida ésta de manera cosificada y como sinónimo en muchas ocasiones de "tradiciones y folclore"). Es por ello que se hace necesario un análisis crítico de dichas políticas que, diseñadas e implementadas a nivel regional, afirman -en el conjunto del Estado- responder a una pretensión de "Educación Intercultural” , pero que en definitiva responden a un modelo de "interculturalidad funcional" (Tubino, 2004) . Dicho análisis tendría que hacerse eco de que la interculturalidad -la "Educación Intercultural”-, en tanto que proyecto político, no puede quedarse en un mero reconocimiento de la diversidad (o de una parte de la misma), sino que ha de propiciar el inicio de procesos de diálogo entre las minorías y la población 
mayoritaria. Ello no buscaría esconder las relaciones y jerarquías de poder, sino más bien visibilizarlas, explicitarlas y ponerlas en el epicentro del diálogo (Quintero et al., 2014), con el objetivo de enfrentar las relaciones de desigualdad existentes en la sociedad. Solo así será posible caminar hacia un proyecto de "interculturalidad crítica" (Freire, 2008; Tubino, 2004; Viaña, 2010; Walsh, 2008) en tanto que "propuesta de sociedad, como proyecto político, social, epistémico y ético dirigido a la transformación estructural (...), asentado en la construcción entre todos de condiciones -de saber, ser, poder y de la vida misma- (...). Pero también como herramienta de accionar; es decir como acción deliberada, constante" (Walsh, 2012, p. 13).

Este trabajo se sitúa en la necesidad de tomar conciencia de esta realidad en el momento de cambio en el que nos encontramos, caracterizado por un endurecimiento de las políticas migratorias a nivel internacional, europeo y español, en particular, a la luz de la presente crisis económico-social, para poder reflexionar sobre la gestión de diversidad lingüístico-cultural en espacios educativos en la actualidad. Los interrogantes de investigación son: 1) ¿Presenta la escuela en el contexto andaluz en la actualidad un elevado grado de multilingüismo gracias a la presencia de alumnado procedente de la inmigración extranjera?; y 2). ¿Están respondiendo las políticas lingüísticas implementadas en espacios escolares a la realidad multilingüe, favoreciendo el acceso en condiciones de igualdad a todo el alumnado a los diferentes itinerarios escolares? Para responder a las mismas los objetivos planteados han sido: por un lado, y con una intención de carácter descriptivo, 1). Mostrar la diversidad lingüística y cultural presente actualmente en la escuela en contexto andaluz, producto de los movimientos migratorios; y por otro lado, 2). Analizar críticamente las políticas educativas que se están implementando para gestionar esta diversidad lingüístico-cultural. A continuación se describe el diseño metodológico seguido para ello.

\section{Método}

El trabajo mostrado en este artículo es fruto de la participación en diversas investigaciones de carácter etnográfico llevadas a cabo por el equipo de investigación del Laboratorio de Estudios Interculturales (Universidad de Granada), del que formo parte como investigadora. Es, por lo tanto, un trabajo que aúna resultados de investigación obtenidos a partir de distintos estudios de carácter cualitativo, con un enfoque eminentemente descriptivo e interpretativo.

En el marco de dichas investigaciones, además de numerosas observaciones y entrevistas que serán descritas a continuación, realizamos un cuestionario sobre diversidad lingüística para conocer sobre algunas de las transformaciones que estaban acarreando los movimientos migratorios internacionales en los espacios educativos. Dicho instrumento, elaborado y puesto en práctica en el curso 2009-10, se utilizó en 9 centros educativos de educación secundaria de 3 ciudades andaluzas . Los ítems sobre los que se preguntó tenían que ver con competencias lingüísticas subjetivas - no se trataba en absoluto de una prueba de nivel- y ámbitos y contextos de uso de las distintas lenguas. Se diseñó con un lenguaje asequible para alumnado de secundaria, y se prestó asesoramiento de traducción en los casos en que fue necesario. En total fueron encuestados 1.953 alumnos/as, entre los 11 y los 19 años. La muestra se compuso de la totalidad del alumnado que cursaba $1^{\circ}, 2^{\circ}, 3^{\circ}$ y $4^{\circ}$ de Educación Secundaria Obligatoria (ESO) de los 9 centros seleccionados, dado que el estudio pretendía explorar la diversidad lingüístico-cultural en los niveles de educación obligatoria superior. El procesamiento estadístico de los datos se hizo a través del paquete informático SPSS .

En cuanto al trabajo de campo etnográfico, realizado de manera casi continuada desde 2004 hasta la actualidad, ha supuesto contar con una gran cantidad de entrevistas, observaciones y grupos de discusión, realizados -insisto- por todo un equipo de investigación. Quiero poner en valor esta cuestión, porque estimo que no es habitual esta 
forma de trabajar realizando etnografía. El trabajo en equipo ha permitido observar 44 centros educativos de secundaria de las distintas provincias de Andalucía. En todos ellos se han realizado entrevistas (121 en total) y observación participante durante los periodos lectivos. Además en 18 de los centros se consiguió realizar grupos de discusión. El trabajo de campo se ha extendido también a las administraciones educativas, habiendo realizado 41 entrevistas a cargos políticos y técnicos de las mismas. El tratamiento de los datos producidos a partir de estas técnicas se ha realizado con el programa informático Nudist Vivo .

Por último se ha realizado una revisión de normativa sobre la materia abordada en este artículo. Ello ha permitido comparar el diseño de las políticas educativas que inciden en la gestión lingüístico-cultural en el contexto abordado, con la implementación de las mismas, observada a partir del trabajo de campo realizado.

\section{Resultados: la diversidad lingüístico- cultural y su tratamiento en la escuela}

Cuando el español es sólo una lengua más

En relación con el primer objetivo planteado en este trabajo, visibilizar la diversidad lingüística del alumnado de los centros considerados, el resultado del análisis del cuestionario apunta a que la escuela andaluza es, en el momento actual, cualquier cosa menos mono-lingue y mono-cultural. Los datos de la Tabla 1 indican las distintas lenguas maternas del alumnado de la muestra, un total de 22 lenguas distintas:

Tabla 1: Lenguas maternas del alumnado entrevistado

\begin{tabular}{lcc}
\hline LENGUA MATERNA & Frecuencia & $\mathbf{\%}$ \\
\hline Árabe (Dariya) & 27 & 1,38 \\
Bereber & 2 & 0,1 \\
Brasileño & 1 & 0,05 \\
Búlgaro & 12 & 0,61 \\
Catalán & 1 & 0,05 \\
Criollo & 1 & 0,05 \\
Chino & 4 & 0,2 \\
Español & 1779 & 91,09 \\
Fang & 1 & 0,05 \\
Fante & 1 & 0,05 \\
Francés & 6 & 0,31 \\
Hindi & 1 & 0,05 \\
Húngaro & 1 & 0,05 \\
Inglés & 56 & 2,87 \\
Italiano & 1 & 0,05 \\
Lituano & 3 & 0,15 \\
Polaco & 2 & 0,1 \\
Portugués & 3 & 0,15 \\
Rumano & 26 & 1,33 \\
Ruso & 9 & 0,46 \\
Ucraniano & 1 & 0,05 \\
Urdu & 2 & 0,1 \\
Perdidos & 13 & 0,67 \\
Total & $\mathbf{1 9 5 3}$ & $\mathbf{1 0 0}$ \\
\hline
\end{tabular}

Como se puede observar, lo más representativo es la gran variedad de lenguas maternas que posee el alumnado encuestado, $\mathrm{y}$ no tanto la cantidad de alumnos/as que habla cada una de ellas. Después del español, la lengua materna más numerosa es el inglés, pero tan solo representa el 2,87\% del total de la muestra, seguido de un $1,38 \%$ del árabe (dariya o árabe marroquí) y de un 1,33\% del rumano. Sin duda son porcentajes muy 
pequeños. Sin embargo cuando consideramos a todo el alumnado cuya primera lengua no es el español, los datos nos dicen que casi un $10 \%$ de la población escolar encuestada tiene como lengua materna una lengua distinta al español, en un sistema educativo (el andaluz) donde el español es la única lengua vehicular oficial. Estos datos han sido respaldados por la información obtenida a través de entrevistas y observaciones. Véase cómo un profesor narraba la "situación lingüística" del centro donde trabajaba:

Hay alumnos de habla inglesa, de Irlanda, que suponen un $3,7 \%(\ldots)$. Y el resto pues según, porque lógicamente hay algunos que hablan español, por ejemplo los de Bolivia o Brasil, estos hablan español, pero tenemos de Israel, India, Polonia, Rusia, Yugoslavia, China (...) y normalmente no saben español, salvo que hayan nacido en España, hay algún chino que ha nacido en España, pero el resto no sabe nada. Hay muchas lenguas, sí. (Profesor de Lengua, 2010).
A continuación, la persona entrevistada, contaba que además entre el alumno chino y el rumano, siendo desconocedores ambos del español, las diferencias son abismales en términos de rapidez de aprendizaje, dada la cercanía/lejanía de sus lenguas maternas con el español. Es importante retener este dato, porque servirá para iniciar el análisis crítico de las políticas educativas de gestión de la diversidad cultural que se muestra en el próximo apartado.

En la Tabla 2 se pueden ver cuales son las segundas lenguas del alumnado encuestado. Casi un 90\% de ellos/as (1.736 alumnos/as) han respondido que hablan una segunda lengua, $\mathrm{y}-\mathrm{a}$ las ya mencionadas como primeras lenguas- se añaden el resto de lenguas co-oficiales del Estado español (euskera, gallego, valenciano), otras lenguas oficiales de países pertenecientes a la Unión Europea (alemán, holandés, italiano) y otras como el bambara, panyabi o wolof. En total, entre lenguas maternas y segundas lenguas, los centros considerados cuentan con 32 lenguas distintas.

Tabla 2: Segunda lengua del alumnado entrevistado

\begin{tabular}{lcc}
\hline SEGUNDA LENGUA & Frecuencia & $\mathbf{\%}$ \\
\hline Alemán & 1 &, 1 \\
Árabe (Dariya) & 29 & 1,5 \\
Bambara & 1 &, 1 \\
Bereber & 1 &, 1 \\
Catalán & 12 &, 6 \\
Chino & 1 &, 1 \\
Español & 106 & 5,4 \\
Euskera & 1 &, 1 \\
Fang & 2 &, 1 \\
Francés & 156 & 8,0 \\
Gallego & 2 &, 1 \\
Holandés & 1 &, 1 \\
Inglés & 1397 & 71,5 \\
Italiano & 6 &, 3 \\
Portugués & 3 &, 2 \\
Panyabi & 1 &, 1 \\
Rumano & 4 &, 2 \\
Ruso & 9 &, 5 \\
Ucraniano & 1 &, 1 \\
Valenciano & 1 &, 1 \\
Wolof & 1 &, 1 \\
Sub-total & 1736 & 88,9 \\
Perdidos & 217 & 11,1 \\
\multicolumn{1}{c}{ Total } & $\mathbf{1 9 5 3}$ & $\mathbf{1 0 0 , 0}$ \\
\hline
\end{tabular}


Las segundas lenguas más habladas son, por orden de frecuencia, inglés, francés y español.

Un 70\% de los/as encuestados/as (1.356 alumnos/as) responde además hablar una tercera lengua (Tabla 3), y hasta un 7\% (142 alumnos/as) de ellos una cuarta lengua. Se añaden a las anteriormente citadas como primeras y segundas lenguas el japonés, el serbio y varias variedades lingüísticas de romaní (usando las categorías emic del propio alumnado “romaní español” y “romaní rumano"). En total, se contabilizaron 36 lenguas distintas en los centros de la muestra considerada.

Tabla 3: Tercera lengua del alumnado entrevistado

\begin{tabular}{lcc}
\hline TERCERA LENGUA & Frecuencia & \% \\
\hline Alemán & 15 &, 8 \\
Árabe (Dariya) & 17 &, 9 \\
Catalán & 10 &, 5 \\
Chino & 1 &, 1 \\
Criollo & 1 &, 1 \\
Español & 38 & 1,9 \\
Euskera & 1 &, 1 \\
Francés & 1038 & 53,1 \\
Gallego & 3 &, 2 \\
Holandés & 1 &, 1 \\
Inglés & 199 & 10,2 \\
Italiano & 13 &, 7 \\
Japonés & 2 &, 1 \\
Portugués & 4 &, 2 \\
Romaní español & 1 &, 1 \\
Romań rumano & 1 &, 1 \\
Rumano & 4 &, 2 \\
Ruso & 2 &, 1 \\
Serbio & 2 &, 1 \\
Ucraniano & 1 &, 1 \\
Valenciano & 1 &, 1 \\
Wolof & 1 &, 1 \\
Total & 1356 & 69,4 \\
Perdidos & 597 & 30,6 \\
Total & $\mathbf{1 9 5 3}$ & $\mathbf{1 0 0}$ \\
\hline
\end{tabular}

Estos datos demuestran el cada vez más patente multilingüismo de las escuelas españolas, y andaluzas, y el plurilingüismo de su alumnado. Quizá la pregunta que cabe realizar ahora es ¿qué nivel de dominio de dichas lenguas manifiesta poseer el alumnado? En el cuestionario realizado se pedía que se respondiese con MUY MAL, MAL, REGULAR, BIEN, MUY BIEN, PERFECTO sobre el dominio poseído de las lenguas habladas, en lo relativo a lectura, expresión oral y expresión escrita. El cuestionario realizado -como se apuntaba más arriba- no se ha basado en una prueba evaluativa de nivel sobre estas cuestiones, sino que lo que interesaba en la investigación eran las valoraciones personales, subjetivas, del alumnado sobre sus competencias lingüísticas. En este sentido los resultados obtenidos han sido los siguientes: 


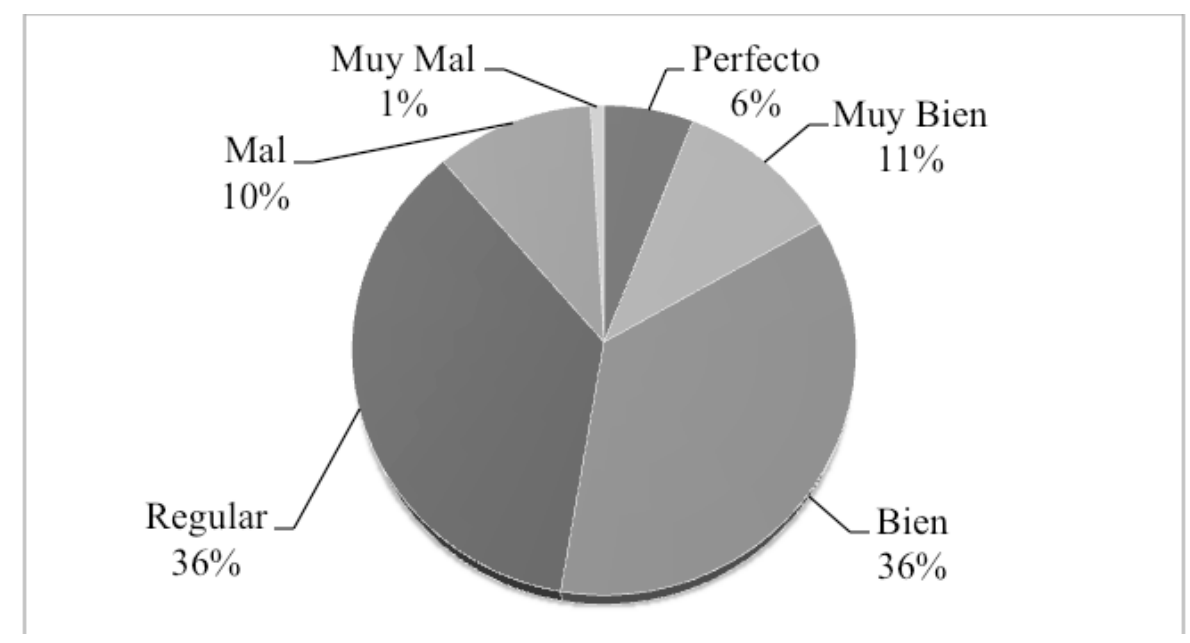

Figura 1: Nivel de competencia de la segunda lengua

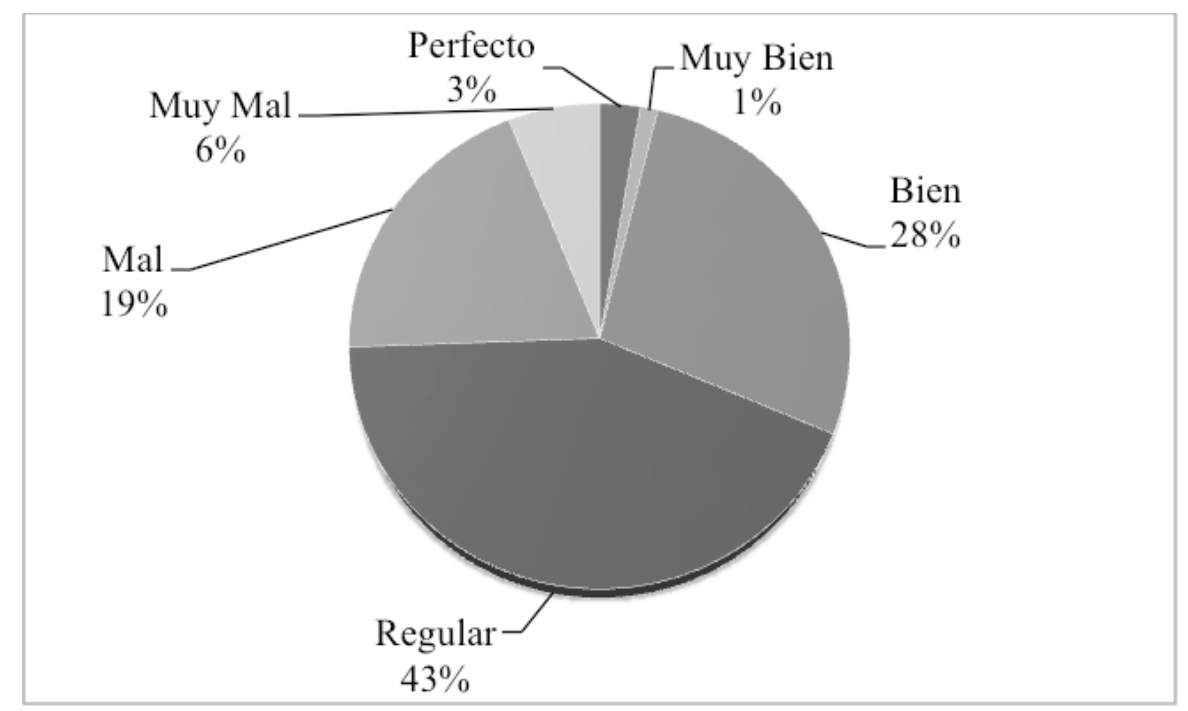

Figura 2: Nivel de competencia de la tercera lengua

En la Figura 1 se puede observar cómo un $6 \%$ del alumnado de la muestra manifiesta hablar PERFECTAMENTE una segunda lengua, más del 10\% MUY BIEN y cerca del $40 \%$ BIEN. Es decir casi el $60 \%$ de la muestra valora positivamente su competencia en la segunda lengua hablada. Con respecto a las terceras lenguas (Figura 2), el nivel disminuye, pero aún así algo más de un 30\% de los/as encuestados/as afirma hablar bien una tercera lengua. Sin embargo el profesorado en muchas ocasiones no percibe esta diversidad como un potencial de los centros si no, muy al contrario, como un hándicap:

Nos lo hemos planteado muchas veces ¿qué formación demandamos? para atender a los alumnos inmigrantes ¿qué hacemos? porque si todos viniesen con el mismo idioma pues podíamos preparar fichas en su idioma, especializándonos nosotros (...) pero si es que aquí hay rumanos, marroquíes, chinos, árabes ¿qué hacemos, qué idiomas aprendemos, cómo?, e incluso para la Delegación es complicado nombrar al profesor de interculturalidad porque ¿a quién nombra? el que venga, cuando no hay conocimiento alguno del idioma, es nulo completamente ¿qué trabajo realizas? (Director de Centro, 2010).

Aludiendo a una "falta de formación” para trabajar en este tipo de situaciones. Una mayor 
dotación de recursos humanos, sin duda, ayudaría también a cambiar la percepción del profesorado en términos de problemática sobre la diversidad en las aulas. Siendo así, recientemente, y en el marco de un grupo de discusión realizado con profesorado de español para alumnado extranjero, personal técnico de las administraciones educativas e investigadores/as especialistas sobre estas temáticas, una profesora, en la línea de lo que afirmaba el director citado, manifestaba lo siguiente:

Nos interesaría saber metodologías, consejos, una forma pedagógica de dar una lengua extranjera, porque no es fácil. Es decir, yo tengo una ligera formación pero hay otros profesores que están enseñando español al alumnado extranjero que no, porque estamos de todo. Estamos profesores de Matemáticas, de Filosofía, de esto de lo otro. O sea, no [tenemos] formaciones específicas. (Profesora de español para extranjeros, 2015).

Quizá lo más llamativo no es la coincidencia en los pareceres de ambos docentes, sino la distancia temporal de cinco años que separa un discurso de otro, lo cual es indicativo de que la inquietud por una mayor y mejor formación se mantiene en el tiempo.

Las políticas educativas de gestión de la diversidad lingüístico-cultural

Con respecto al segundo objetivo planteado en este artículo, analizar las políticas educativas diseñadas para gestionar la diversidad lingüístico-cultural, es necesario retomar la idea esbozaba más arriba sobre la importancia de tomar en consideración el porcentaje de alumnado de nuestra muestra que no tiene el español como lengua materna: casi un $10 \%$. Ello es significativo porque las políticas de gestión de la diversidad cultural en el país, y en Andalucía, son esencialmente políticas de gestión de la diversidad lingüística. Dicho con otras palabras, cuando se observa cómo es el tratamiento otorgado a la población escolar identificada como “inmigrante" en España se ve cómo los planes de acogida están consistiendo fundamentalmente en la elaboración de dispositivos específicos destinados a atender a quienes no dominan la lengua vehicular de la escuela (Olmos-Alcaraz, 2010, 2012; García et al., 2011). En el caso de Andalucía, el español.

A pesar de esto los centros educativos "no cuantifican" lenguas para solicitar a la administración educativa recursos para implementar programas específicos. Ello lleva a una primera consideración crítica sobre las políticas educativas de gestión de la diversidad lingüístico-cultural, dado que no es posible conocer de manera exhaustiva sobre diversidad lingüística si lo que observamos es diversidad de nacionalidades (que es lo que se cuantifica en las estadísticas escolares). Veamos cómo se confunde esto en parte del profesorado, incluso cuando -aparentementese conocen "las diferencias". En este caso el Jefe de Estudios de un centro educativo asimila "inmigrante" a "hijo de inmigrare", cuando narra cómo la administración educativa proporciona recursos a su centro:

Sabemos enfrentarnos al problema de cuando un niño tiene un nivel cero de comunicación, a parte de que ya la Delegación también nos ha mandado recursos, tenemos un profesor para esos niños, porque ahora mismo tenemos 76 alumnos inmigrantes, ahora mismo hay 76 alumnos hijos de inmigrantes Nosotros distinguimos entre inmigrantes y extranjeros te voy a decir por qué. Muchos de los niños que ya van naciendo aquí en España, ya toman la nacionalidad española entonces si tú miras la nacionalidad pues solamente hay me parece que son 10 países diferentes, pero si tu miras por otra parte la procedencia de nuestros alumnos, pues proceden de 12 países diferentes. Entonces ahí hay una diferencia, nosotros hacemos esa distinción (...). Estos alumnos hijos de inmigrantes están aquí, hay 35 de Marruecos, 6 de Colombia, 15 de Ecuador, 7 de Portugal, 1 de Croacia, 2 senegaleses, 3 argelinos, 
2 bolivianos, una polaca, una argentina...

- (Jefe de Estudios, 2010).

Y veamos además cómo difieren los datos en la muestra del cuestionario realizado. En la
Tabla 4 se puede observar una relación de las lenguas maternas habladas por el alumnado en función de la nacionalidad que ostenta, aunque distingue solo entre nacionales y extranjeros.

Tabla 4: Lenguas maternas del alumnado entrevistado por nacionalidad

\begin{tabular}{lccc}
\hline \multirow{2}{*}{ LENGUA MATERNA } & \multicolumn{2}{c}{ NACIONALIDAD } & \multirow{2}{*}{ Total } \\
\cline { 2 - 3 } Árabe (Dariya) & Española & Extranjera & $27(1,4 \%)$ \\
\cline { 2 - 3 } Bereber & $4(, 2 \%)$ & $23(7,6 \%)$ & $2(, 1 \%)$ \\
Brasileño & $0(, 0 \%)$ & $2(, 7 \%)$ & $1(, 1 \%)$ \\
Búlgaro & $0(, 0 \%)$ & $1(, 3 \%)$ & $12(, 6 \%)$ \\
Catalán & $0(, 0 \%)$ & $12(4,0 \%)$ & $1(, 1 \%)$ \\
Criollo & $1(, 1 \%)$ & $0(, 0 \%)$ & $1(, 1 \%)$ \\
Chino & $0(, 0 \%)$ & $1(, 3 \%)$ & $4(, 2 \%)$ \\
Español & $0(, 0 \%)$ & $4(1,3 \%)$ & $1776(91,2 \%)$ \\
Fang & $1574(95,7 \%)$ & $202(66,7 \%)$ & $1(, 1 \%)$ \\
Fante & $0(, 0 \%)$ & $1(, 3 \%)$ & $1(, 1 \%)$ \\
Francés & $0(, 0 \%)$ & $1(, 3 \%)$ & $6(, 3 \%)$ \\
Hindi & $4(, 2 \%)$ & $2(, 7 \%)$ & $1(, 1 \%)$ \\
Húngaro & $0(, 0 \%)$ & $1(, 3 \%)$ & $1(, 1 \%)$ \\
Inglés & $0(, 0 \%)$ & $1(, 3 \%)$ & $55(2,8 \%)$ \\
Italiano & $52(3,2 \%)$ & $3(1,0 \%)$ & $1(, 1 \%)$ \\
Lituano & $0(, 0 \%)$ & $1(, 3 \%)$ & $3(, 2 \%)$ \\
Polaco & $0(, 0 \%)$ & $3(1,0 \%)$ & $2(, 1 \%)$ \\
Portugués & $1(, 1 \%)$ & $1(, 3 \%)$ & $3(, 2 \%)$ \\
Rumano & $0(, 0 \%)$ & $3(1,0 \%)$ & $26(1,3 \%)$ \\
Ruso & $1(, 1 \%)$ & $25(8,3 \%)$ & $9(, 5 \%)$ \\
Ucraniano & $0(, 0 \%)$ & $93,0 \%()$ & $1(, 1 \%)$ \\
Urdu & $0(, 0 \%)$ & $1(, 3 \%)$ & $2(, 1 \%)$ \\
Perdidos & $0(, 0 \%)$ & $2(, 7 \%)$ & $12(, 6 \%)$ \\
\hline Total & $8(, 5 \%)$ & $4(1,3 \%)$ & $\mathbf{1 9 4 8}(100,0 \%)$ \\
\hline
\end{tabular}

Algo más de $5 \%$ de los estudiantes españoles no tienen como lengua materna el español, y -lo que es más interesante- casi un $67 \%$ de los extranjeros tienen como lengua materna el español. Entre los primeros estarán hijos de parejas mixtas (español/aextranjero/a), hijos de familias inmigradas que ya hayan nacido en España (las llamadas "segundas generaciones"), o españoles cuyas lenguas maternas sean las co-oficiales del Estado español, entre otras casuísticas; entre los segundos estarán fundamentalmente todos aquellos/as alumnos/as extranjeros procedentes de países donde se habla español.

Estos datos indican que la nacionalidad no es un predictor efectivo para justificar la implementación o no de una política educativa de gestión de la diversidad lingüística, por razones, a priori, bastante obvias: una nacionalidad no es igual a una lengua. No obstante en el contexto español se cuenta, ya desde hace algún tiempo, con estadísticas sobre extranjería en contextos escolares, pero no con estadísticas nacionales o regionales sobre diversidad lingüística relacionada con la 
llegada de población inmigrante al país, que pudiesen ofrecer datos no solo de cuántos alumnos no cuentan con el español como lengua materna, sino cuántos de ellos conviven en situaciones familiares multilingües y, sobre todo, de qué lenguas se trata.

A continuación se analizan dos políticas educativas de gestión de la diversidad lingüística bien diferenciadas en el contexto andaluz. Ambas forman parte de lo que se está construyendo a nivel político como “Educación Intercultural”. Por un lado, y con una antigüedad mayor en el tiempo encontramos el Plan Andaluz de Educación de Inmigrantes (desde 2001), en adelante PAEI. $\mathrm{Y}$ en segundo lugar, desde el año 2005, se implementa también el Plan de Fomento del Plurilingüismo, en adelante PFP. Ambos planes contemplan entre sus objetivos la pretensión de interculturalizar los centros educativos:

Objetivos del Plan Andaluz de Educación a Inmigrantes:

\section{Objetivo $N^{o} 2$.}

Favorecer que los centros elaboren Proyectos Educativos interculturales que faciliten y promuevan procesos de intercambio, interacción y cooperación entre las culturas (Plan Andaluz de Educación a Inmigrantes 2001, p. 12).

Objetivos específicos del Plan de Fomento del Plurilingüismo:

[...] Se potenciará la educación intercultural dirigida al conjunto del alumnado con el objeto de incrementar la sensibilización intercultural, promover la tolerancia e implantar el respeto mutuo entre el alumnado de distintos orígenes lingüísticos y culturales. En este sentido se propondrá a la administración central la incorporación de nuevas lenguas como primer idioma, especialmente las más habladas por nuestro alumnado inmigrante (Plan de Fomento del Plurilingüismo, 2005, p. 56).

Pero ninguno de ellos lo está consiguiendo, como a continuación se pasa a exponer.
Consideremos la definición de "Educación Intercultural" que realiza el gobierno autonómico, dado que hay que ser conscientes de la existencia de una gran variedad de modelos y tipologías de la misma (García et al., 2011; Dietz, 2012,). Siendo así, desde la administración se realiza la siguiente definición de "Educación Intercultural”:

La perspectiva intercultural parte de un planteamiento donde lo sustantivo es la interacción, el reconocimiento de que lo cultural es necesariamente un fenómeno interactivo donde no es posible poner barreras. Construir la interculturalidad precisa de la posibilidad de afirmar la propia cultura en su relación con las otras culturas. Esto sólo es posible si se establece un proceso donde todas $\mathrm{y}$ todos puedan aportar $\mathrm{y}$ donde estas aportaciones sean sujeto de intercambio $y$ valoración crítica (...). La interculturalidad obliga a pensar en las relaciones culturales dentro de un proyecto educativo, pero también dentro de un proyecto social, que supone hacer posible la igualdad de derechos $\mathrm{y}$ de oportunidades entre los seres humanos que conviven en una sociedad determinada (Plan Andaluz de Educación a Inmigrantes, 2001, p. 8-9).

Entendiendo por la misma un enfoque que promueva la interacción de todos los grupos presentes en la escuela; y un proyecto que ha de ser educativo y social, es decir, tener presencia dentro y fuera de la escuela. En la aplicación práctica de ambos planes, sin embargo, lo que más destaca es la diferenciación entre distintos tipos de alumnado, consiguiendo justamente lo contrario de lo que promulga: la no interacción, el no diálogo, la no convivencia. Veamos por qué.

En el marco del PAEI la acción que más destaca es sin duda la creación de las Aulas Temporales de Adaptación Lingüística (en adelante ATAL), dispositivos pensados para enseñar español al alumnado recién llegado a España. Desde 2007 se cuenta con un desarrollo normativo para ello, a través de la Orden de 15 de enero de 2007, por la que se 
regulan las medidas y actuaciones a desarrollar para la atención del alumnado inmigrante. No obstante dicha Orden dedica 11 de sus 13 artículos a cuestiones que tienen que ver exclusivamente con la enseñanza del español. Los otros 2 artículos restantes se dedican a apuntar medidas de acogida para el alumnado inmigrante (art. 3) y medidas para el mantenimiento de la cultura de este (art. 13).

Es decir, nos encontramos con que el desarrollo normativo de lo que debiera ser un gran pilar de la "Educación Intercultural” en el contexto estudiado se centra de manera muy importante en cuestiones lingüísticas circunscritas a parte de la población inmigrante extranjera: enseñar español. ¿Qué pasa entonces con el presupuesto de una "Educación Intercultural" como un proyecto donde "todos puedan aportar y donde estas aportaciones sean sujeto de intercambio y valoración crítica” (PAEI, 2001, p. 9)? Desde luego con este tipo de medidas parece estar fomentándose más la asimilación lingüística que el plurilingüismo y el multilingüismo. Pero veamos qué aportaciones hace la segunda política mencionada, el PFM.

En este caso, a pesar de que uno de sus objetivos -como se reseñaba- era "la incorporación de nuevas lenguas como primer idioma, especialmente las más habladas por nuestro alumnado inmigrante” (PFP, 2005, p. 56), los programas existentes no responden exactamente a ello. En el marco del PFM se han creado en Andalucía desde 2005, 12 centros bilingües español-alemán, 59 centros bilingües español-francés y 782 centros bilingües español-inglés (datos para el curso 2013-14). Por lo tanto, las lenguas de los "inmigrantes", y sobre todo de los “inmigrantes no europeos” están ausentes de este plan. Se ha de insistir en que una nacionalidad no es igual a una lengua, pero ello no impide que podamos observar cómo la nacionalidad extranjera en la escuela española más numerosa sea la marroquí, con un 19,8\% del alumnado, no la británica, la estadounidense, la francesa o la alemana, por ejemplo.
Reflexionemos también a cerca de quienes son los/as alumnos/as que cursan los programas bilingües mencionados. La crítica anterior quería reflejar una ausencia de ciertas lenguas en los programas de plurilingüismo del contexto estudiado, y que evidencian las contradicciones entre el diseño teórico de la normativa y la implementación de la misma . No obstante podríamos pensar que el PFP está priorizando el bilingüismo con lenguas que son competitivas en el mercado laboral actual, y que serán necesarias y muy útiles para el desempeño profesional futuro del alumnado. Este argumento podría servir perfectamente para el inglés; pero no está tan claro para francés y mucho menos para alemán. Con esta misma lógica cabría preguntarse ¿por qué no se enseña chino pensando en la potencialidad que ello puede conllevar en un futuro no muy lejano debido a la cada vez mayor emergencia de empresas chinas a nivel internacional? Sin embargo es mucho más problemático observar quienes están accediendo a estos programas bilingües. Tampoco en este caso se cuenta con estadísticas al respecto, pero mi práctica basada en la realización de trabajo de campo etnográfico durante un periodo prolongado de tiempo -tal y como se menciona en el apartado metodológico de este artículo- permite afirmar que no son los/as alumnos/as inmigrantes de nacionalidad extranjera, ni los alumnos descendientes de familias migrantes, quienes en mayor medida acceden a los programas bilingües. Así lo expresaba una profesora entrevistada: "Los inmigrantes no suelen estar porque el profesorado en muchas ocasiones recomienda ya a quienes ven como mejores alumnos que cursen el itinerario bilingüe. Los inmigrantes suelen tener peores notas, no suelen ser los mejores estudiantes, y por lo tanto no suelen estar en este programa, no" (Profesora de Música, 2012). Estos/as, mientras sus compañeros/as españoles/as e hijos/as de españoles/as se insertan en dichos programas, salen del aula normalizada para ir a clase de español, o a diversificación curricular o algún tipo de programa de atención educativa especial . 
Es decir, mientras un alumnado es atendido en términos de interculturalidad con el PAEI, otro, con el mismo argumento lo es a través del PFP. Y ello teniendo en cuenta que muchos de estos/as alumnos/as procedentes de la inmigración ya tienen como segunda lengua precisamente inglés o francés, debido a la escolarización previa en países donde en muchas ocasiones la lengua vehicular de la escuela es la lengua de la antigua metrópoli.

\section{Discusión}

Existe una gran diversidad lingüística -y por ende cultural- en las aulas andaluzas, en tanto que exponente de la situación actual en España debido a los movimientos migratorios internacionales de las últimas décadas. En este contexto, y con la emergencia y desarrollo del discurso intercultural en el país, el diseño de políticas educativas con objetivos que apuntan a querer gestionar la diversidad se está dibujando -por el contrario- con tintes diferenciadores.

En la muestra considerada se evidencia una composición del alumnado marcadamente heterogénea y plurilingüe, lo que podría ser aprovechado para construir espacios paradigmáticos de multilingüismo e interculturalidad. No obstante el camino andado hasta el momento presenta importante escollos, no desmereciendo con esta afirmación los esfuerzos volcados en la labor educativa por los/as profesionales implicados en la gestión e implementación de los planes comentados en este artículo . Siendo así, y a modo de conclusiones propositivas, habría de considerarse que:

- Es preciso reconocer institucionalmente la diversidad lingüística presente en las aulas, con políticas educativas integrales de corte intercultural. En otras palabras, la educación no puede segregar si se quiere que sea intercultural. Los mayores problemas que han surgido de programas educativos interculturales en diversos contextos tienen que ver precisamente con una no correspondencia con lo diseñado a nivel teórico-político y lo implementado finalmente sobre el terreno (Muñoz, 2009), o con la asunción de un paradigma intercultural funcional (Tubino, 2011; Walsh, 2012) que no responde a las necesidades y características de las sociedades donde se aplica. Ello es así también en el caso de Andalucía, de España, en lo relativo a esta cuestión: redactados de leyes, políticas y planes recogen unas definiciones de "Educación Intercultural" acordes con la idea de un intercambio igualitario entre los distintos grupos implicados que no segrega, pero no llegan a implementarse como tal. Y el profesorado lleva tiempo demandando mayores recursos y formación para atender de manera adecuada la realidad a la que se enfrenta todos los días. Los objetivos propuestos por las instancias gubernamentales del contexto estudiado son bastante ambiciosos, loables si se quiere, justos si tomamos conciencia de la composición actual de población. Pero, como se ha mostrado a lo largo del texto, no basta con la formulación teórica de unos objetivos si la traducción práctica de los mismos apuntan justamente en dirección contraria; o si no se consideran de manera adecuada las demandas de quienes, en definitiva, están atendiendo en la escuela día a día estas diversidades. Es necesario por ello impulsar proyectos educativos verdaderamente plurilingües asentados en un conocimiento efectivo de la diversidad en términos lingüísticos, pero que no olvide las otras dimensiones de la mismaque den respuesta a los contextos concretos en los que se aplican y a las heterogeneidades específicas que estos albergan.

- Numerosos estudiosos han indicado ya las ventajas que supone una educación propiciada en distintos idiomas (Martín \& Mijares, 2007; García-Cano et al., 2010; Siqués \& Vila, 2014). Para el caso del alumnado inmigrante, la creación de programas formativos adecuados en las respectivas lenguas maternas ayudan a una mejor adquisición de una segunda lengua (Cummins, 1979; Hamel, 1995; Vila, 2000). En el contexto observado, Andalucía, una investigación realizada recientemente sobre las diferencias entre alumnado que cursa la línea bilingüe $\mathrm{y}$ 
alumnado que no lo hace, revela la obtención de mejores resultados de formación en quienes asisten al grupo bilingüe, porque mejoran sus formas de estudiar, tienen mejor uso de las nuevas tecnologías y mejores hábitos de lectura (Caballero \& Reyes, 2011). En este sentido otra de las recomendaciones sería que se trabajase para que los/as alumnos/as inmigrantes extranjeros/as estuviesen más presentes en los programas bilingües, dados los notables beneficios que -para toda la comunidad educativa - se derivan de ello. Se trata de alumnos/as que, en su mayoría, proceden de países con pasados coloniales en los que en la escuela se habla otra lengua distinta a sus lenguas maternas, y en muy alto grado se trata de francés o inglés (las dos lenguas más representativas de los programas bilingües en Andalucía). Es decir, es alumnado que domina más de una lengua, pero la escuela parece entender esta característica más como un hándicap que como una oportunidad, derivando a los mismos a los programas de compensatoria.

- El modelo de gestión de la diversidad lingüística debería caminar hacia el multilingüismo y no hacia el bilingüismo. Se podrían incluir las lenguas maternas del alumnado inmigrante entre las opciones posibles de los programas bilingües. Con ello, además de trabajar para conseguir la no diferenciación del alumnado y/o exclusión de parte de este, se estaría propiciando el mantenimiento de la diversidad presente actualmente las aulas. Ello redundaría, como ya se ha visto en otros contextos especialmente relativos a países latinoamericanos- en un re-descubrimiento, reconocimiento y valoración de las heterogeneidades, generando diálogo entre sujetos con adscripciones culturales distintas y contribuyendo al desarrollo pleno de las identidades (interculturales) de todos los estudiantes (Quilaqueo \& Quintriqueo, 2008). Desde luego todo ello requiere de una inversión de recursos, humanos y económicos, en los centros educativos que debe tenerse muy presente en un contexto de crisis económica como en el que estamos inmersos.
- Por último insistir, como ya lo han hecho gran cantidad de investigaciones llevadas a cabo por todo el país (y a nivel internacional), en la necesidad de una formación -inicial y continua- en interculturalidad para trabajadores de las administraciones públicas en general (Donoso, Cabrera, Aneas, De Santos \& Curós, 2009; Martínez, 2011); y en especial sobre "Educación Intercultural" para los/as docentes (Aguado, Gil \& Mata, 2010; Soriano \& González, 2010; Soriano \& Peñalva, 2011; Leiva, 2012). Ello aportaría herramientas críticas para diseñar y desarrollar en los respectivos contextos de trabajo enfoques, programas y planes educativos acordes con la diversidad presente en las aulas. No se trata, sin embargo, de "conocer todas las lenguas" -como apuntaba uno de los profesores citados más arriba-, sino de dotar al profesorado de habilidades y destrezas orientadas hacia la crítica y la transformación social (Quintero et al., 2014). En otras palabras, de formar con y en competencias interculturales (Olmos-Alcaraz \& Martínez, 2014) que propicien la emergencia de procesos de innovación y cambio desde los/as propios docentes. Para ello trabajar desde una perspectiva interseccional (Crenshaw, 1991) podría dar claves para una mejor gestión de la diversidad en las aulas. Considerar la existencia de diferentes ejes alrededor de los que giran las categorías que generan procesos de desigualdad y prescindir de análisis que entienden la misma como una consecuencia lineal de las culturas de origen y las lenguas maternas del alumnado, proporcionaría herramientas para abordar la gestión de la diversidad en las aulas de manera más holista y contextualizada (Olmos-Alcaraz \& Rubio, 2013, 2014).

\section{References}

Aguado, M.T., Gil, I. \& Mata, P. (2010). El enfoque intercultural en la formación del profesorado. Dilemas y propuestas. Contrastes: Revista cultural, 58, 11-19.

Alegre, M.A. (2011). Educación e inmigración. ¿Un binomio problemático? En F.J. García \& S. Carrasco (Eds.), Población inmigrante y 
escuela: conocimientos y saberes de investigación (pp. 339-364). Madrid: Ministerio de Educación.

Antolínez, I. \& García-Cano, M. (2011). Miradas antropológicas en clave intercultural hacia una escuela que decide ser comunidad de aprendizaje. En F. Villalba \& J. Villatoro (Eds.). Educación Intercultural y Curriculum (Vol. II) (pp. 130-143). Málaga: Letra 25.

Caballero, M. G. \& Reyes, M. (2011). El nuevo alumnado surgido del plan de fomento del plurilingüismo de la Junta de Andalucía. España. Estudio comparativo entre alumnos de $4^{\circ}$ de la ESO. Revista Fuentes, 11, 139-160. Consultado en http://institucional.us.es/revistas/fuente/11/art_ 7.pdf

Cummins, J. (1979). Linguistic Interdependence and the educational development of bilingual children. Review of Educational Research, 2(49), 222-251. doi: https://doi.org/10.3102/00346543049002222

Carrasco, S. (2003). La escolarización de los hijos e hijas de inmigrantes y de minorías étnico-culturales. Revista de Educación, 330, 99-136. Consultado en http://www.mecd.gob.es/dctm/revista-deeducacion/articulosre330/re3300611213.pdf?d ocumentId=0901e72b81258ccc

Carrasco, S. (2011). Interculturalidad e inclusión: principios para evaluar la acogida al alumnado de origen extranjero. En F.J. García \& S. Carrasco (Eds.), Población inmigrante y escuela: conocimientos y saberes de investigación (pp. 493-502). Madrid: Ministerio de Educación.

Castilla, J. (2014). Las ATALs: ¿Integración o segmentación vertical? En C. Peláez \& M. I. Jociles (Eds.), Estudios etnográficos de políticas públicas en contextos educativos (pp. 57-66). Madrid: Traficantes de Sueños.

Contini, P., Olmos-Alcaraz, A. \& Contini, P. (2016). Managing the Cultural Differences in Spain: When Talking about Interculturality Means Talking about Immigrant's Culture. Mediterranean Journal of Social Science, 7
(1),

63-73.

doi:

https://doi.org/10.5901/mjss.2016.v7n1s1p63

Cucalón, P. (2014). Tránsitos, límites y migrantes en las escuelas. Una investigación en las Aulas de Enlace de la Comunidad de Madrid (Tesis doctoral). Madrid: Universidad Complutense de Madrid.

Crenshaw, K. (1991). Mapping the margins: intersectionality, identity politics and violence against women of color. Stanford Law Review, 43(6), 1241-1299. doi: https://doi.org/10.2307/1229039

Del Olmo, M. \& Hernández, C. (2004). Diversidad cultural y educación: la perspectiva antropológica en el análisis del contexto escolar. En M. I. Vera \& D. Pérez (Coords), Formación de la ciudadanía: las TICs y los nuevos problemas (1-13). Alicante: AUPDCS. Consultado

en http://www.uned.es/grupointer/art_olmo_herna ndez_div_edu_04.pdf

Del Olmo, M. (2012). Buenas prácticas, ¿desde el punto de vista de quién? Una contribución a la controversia sobre las aulas de enlace. Revista de Educación, 358, 111-128. doi: http://doi.org/10-4438/1988-592X-RE-2012358-185

Dietz, G. (2012). Multiculturalismo, Interculturalidad y diversidad en educación: una aproximación antropológica. México: Fondo de Cultura Económica.

Donoso, T., Cabrera, F.A., Aneas, A., De Santos, J. \& Curós, P. (2009). Análisis de necesidades en formación intercultural en la administración pública. Revista de Investigación Educativa, 27(1), 149-167. Consultado en http://revistas.um.es/rie/article/download/9434 $\underline{1 / 102971}$

Fernández, J. y García, F.J. (2015). El desarrollo normativo que regula las aulas escolares de nacionalidad extranjera. Profesorado. Revista de currículum y formación del profesorado, 19(1), 468-495. Consultado en http://www.ugr.es/local/recfpro/rev191COL11. pdf 
Franzé, A. (2003). Imágenes y prácticas de los usos de la interculturalidad en el campo educativo. En A. Barañano \& J. L. García (Coords.), Culturas en contacto: encuentros y desencuentros (pp. 271-278). Madrid: Ministerio de Educación, Cultura y Deporte.

Freire, P. (2008). Pedagogía del oprimido. Madrid: Siglo XXI.

García, F. J., Fernández, J., Rubio, M. \& Soto, L. (2011). Inmigración extranjera y educación en España: algunas reflexiones sobre el “alumnado de nueva incorporación”. En F.J. García \& S. Carrasco (Eds.), Población inmigrante y escuela: conocimientos y saberes de investigación (pp. 403-492). Madrid: Ministerio de Educación.

García, F. J. \& Martínez, A. (2000). ¿Qué hay de intercultural en las acciones interculturales? El caso de la atención a los inmigrantes extranjeros. Qurriculum,14, 9-27. Consultado en $\quad$ http://ldei.ugr.es/javiergarcia/wpcontent/uploads/2012/10/GarciaGranados2000. pdf

García, F. J. y Olmos-Alcaraz, A. (2010). La promoción de la plena integración de los extranjeros por parte de las administraciones públicas: ¿una protección legal para la construcción de una sociedad multicultural?. En J. L. Monereo (dir.), Protección jurídicosocial de los trabajadores extranjeros, Colección Trabajo y Seguridad Social, (p. 111-177). Granada: Comares S.L.

García, F.J \& J. Ortega (Eds.) (2015). Actas del VIII Congreso sobre Migraciones Internacionales en España (Granada, 16-18 de septiembre de 2015). Granada: Instituto de Migraciones.

García-Cano, M., González, E. M., Márquez, E., Dietz, G., Pozo, M. T. \& Muriel López, C. (2010). Estrategias bilingües e interculturales en familias transmigrantes. Revista de Educación, 352, 289-308. Consultado en http://www.revistaeducacion.educacion.es/re35 2/re352_13.pdf

Hamel, R.E. (1995). Conflictos entre lenguas y derechos lingüísticos: perspectivas de análisis sociolingüístico. Alteridades, 10(5), 79-88. Consultado en http://www.uamantropologia.net/pdfs/ceida/alte_10_7.pdf

Jiménez, R. (2012). Diversidad cultural y lingüística, identidad e inmigración: algunas conclusiones y propuestas desde la investigación educativa. Revista de Educación Inclusiva, 5(1), 139-156. Consultado en http://www.ujaen.es/revista/rei/linked/docume ntos/documentos/15-11.pdf

Junta de Andalucía (1999). Ley andaluza 9/1999, de 18 de noviembre, de Solidaridad en la Educación. Consultado en http://www.juntadeandalucia.es/boja/1999/140 $\underline{11}$

Junta de Andalucía (2001). Plan Andaluz de Educación a Inmigrantes. Sevilla: Junta de Andalucía.

Junta de Andalucía (2002, 2006, 2014). Plan Integral para la Inmigración en Andalucía. Sevilla: Junta de Andalucía.

Junta de Andalucía (2002). Plan de Cultura de Paz y no Violencia. Consultado en http://www.juntadeandalucia.es/educacion/port al/com/bin/Contenidos/PSE/participacion/Cult uradepaz/PlanAndaluzCulturaPazyNoviolencia /1147779791021_plan_cultura.pdf

Junta de Andalucía (2003). Decreto 167/2003, por el que se establece la ordenación educativa a los alumnos y alumnas con necesidades educativas especiales asociados a condiciones sociales desfavorecidas. Consultado en http://www.juntadeandalucia.es/boja/2003/118 /d3.pdf

Junta de Andalucía (2005). Plan de Fomento del Plurilingüismo: Una política lingüística para la sociedad andaluza. Consultado en http://www.juntadeandalucia.es/averroes/averr oes/html/portal/com/bin/contenidos/B/Innovaci onEInvestigacion/ProyectosInnovadores/Plurili nguismo/Portada/1182945265640_wysiwyg_pl an.pdf

Junta de Andalucía (2007). Orden de 15 de enero de 2007, por la que se regulan las medidas y actuaciones a desarrollar para la 
atención del alumnado inmigrante $y$, especialmente, las Aulas Temporales de Adaptación Lingüística. Consultado en http://www.juntadeandalucia.es/boja/2007/33/ $\underline{\mathrm{d} 1 . p d f}$

Junta de Andalucía (2013). La educación en Andalucía. Curso 2013-14. Iniciativas, programas y datos. Sevilla: Junta de Andalucía.

Leiva, J.J. (2012). La formación en interculturalidad del profesorado y la comunidad educativa. Revista Electrónica de Investigación y Docencia, Monográfico Octubre 2012, 8-31. Consultado en http://www.ujaen.es/revista/reid/monografico/ n2/REIDM2art1.pdf

Márquez, E. \& García-Cano, M. (2014). Condiciones de posibilidad y desarrollo para una educación intercultural crítica. Tres estudios de caso en el contexto. Reis: Revista española de investigaciones sociológicas, 148, 157-170.

doi:

https://doi.org/10.5477/cis/reis.148.157

Martín, L. \& Mijares, L. (2007). "Sólo en español": una reflexión sobre la norma monolingüe y la realidad multilingüe en los centros escolares. Revista de Educación, 343, 93-112. Consultado en http://www.revistaeducacion.mec.es/re343_05. $\underline{\mathrm{html}}$

Martínez, R. (2011). Aprendiendo a gestionar la diversidad cultural en las administraciones públicas. En F.J. García \& N. Kressova (Coords), Actas del I Congreso Internacional sobre Migraciones en Andalucía (pp. 753760). Granada: Instituto de Migraciones.

Meissner, F. \& Vertovec, S. (2015). Comparing Super-diversity. Ethnic and Racial Studies 38(4), 541-555. doi: https://doi.org/10.1080/01419870.2015.980295

Muñoz, H. (2009). Comunidades lingüísticas e identidades colectivas minorizadas: de la protección y del reconocimiento a los derechos culturales. Confluenze. Revista di Studi Iberoamericani, 1(1), 220-239. doi: http://doi.org/10.6092/issn.2036-0967/1426
Olmos-Alcaraz, A. (2010). Construcción discursiva del alumno inmigrante extranjero en Andalucía: el otro en nuestras escuelas. Revista de Educación, 353, 469-493.

Olmos-Alcaraz, A. (2010b). Informe estadístico sobre la situación lingüística en escuelas de Andalucía (Proyecto KALECO: langues en couleurs). Multicopiado.

Olmos-Alcaraz, A. (2012). Cuando migrar se convierte en estigma: un estudio sobre construcción de alteridad hacia la población inmigrante extranjera en la escuela. Imagonautas. Revista Interdisciplinaria Sobre Imaginarios Sociales, 1(2), 62-85.

Olmos-Alcaraz, A. \& Contini, P. (2016). Las ausencias del paradigma intercultural en España en contextos urbanos multiculturales. Revista Mexicana de Sociología, 78(4), 685711.

Olmos-Alcaraz, A. \& Martínez, R. (2014). Plataformas virtuales e innovación docente universitaria: Affordance de una Guía de Trabajo Autónomo en Antropología para trabajar competencias de intervención en contextos de diversidad. Revista de Educación a Distancia, 42, 1-15.

Olmos-Alcaraz, A. \& Rubio, M. (2013). Corporalidad del $<<$ buen estudiante $>>$ : representaciones de género, “raza”, etnia y clase social en la escuela española. Revista Iberoamericana de Educación, 63, 163-179.

Olmos-Alcaraz, A. \& Rubio, M. (2014). Imaginarios sociales sobre "la/el buen y la/el mal estudiante": sobre la necesidad de un análisis interseccional para entender las lógicas de construcción de la diferencia hacia el alumnado “inmigrante”. En P. Cucalón (ed.), Etnografía de la escuela y la interseccionalidad, (p. 9-15). Madrid: Traficantes de Sueños.

Ortiz, M., (2005). Alumnado extranjero en el sistema educativo andaluz. Racialismo en el discurso y práctica escolar (Tesis doctoral). Granada: Universidad de Granada.

Padilla, B., Azevedo, J. \& Olmos-Alcaraz, A. (2015). Superdiversity and conviviality: 
exploring frameworks for doing ethnography in Southern European intercultural cities. Ethnic and Racial Studies, 38(4), 621-635. doi: https://doi.org/10.1080/01419870.2015.980294

Poveda, D., Jociles, M.I. \& Franzé, A. (2009). La diversidad cultural en la educación secundaria en Madrid: Experiencias y prácticas institucionales con alumnado inmigrante latinoamericano. Papeles de Trabajo sobre Cultura, Educación y Desarrollo Humano, 5(3), 1-42. Consultado en http://www.uam.es/otros/ptcedh/2009v5_pdf/v 5n3esp.pdf

Rodríguez, H., Gallego, B., Sansó, C., Navarro, J.L., Velicias, M. \& Lago, M. (2011). La educación intercultural en los centros escolares españoles. REIFOP, 14(1), 101-112. Consultado en http://www.aufop.com/aufop/uploaded_files/ar ticulos/1301588174.pdf

Quilaqueo, S. \& Quintraqeo, S. (2008). Formación docente en educación intercultural para contexto mapuche en Chile. Cuadernos interculturales, 6(10), 91-110.

Quintero, S., Quilaqueo, D., Lepe-Carrión, P., Riquelme, E., Gutiérrez, M. \& Peña-Cortés, F. (2014). Formación del profesorado en educación intercultural en América Latina. El caso de Chile. Revista electrónica interuniversitaria de formación del profesorado, 17(2), 201-217.

Rubio, M. (2013). Construyendo diferencias desde retóricas de la igualdad. El caso del alumnado denominado latinoamericano en la etapa de educación secundaria obligatoria (Tesis Doctoral). Granada: Universidad de Granada.

Sánchez, P. \& García, R. (2011). Organización de las aulas de enlace en la Comunidad de Madrid. Cultura y Educación: Revista de teoría, investigación y práctica, 23(1), 129139. doi: https://doi.org/10.1174/113564011794728614

Santamaría, E. (2002). Inmigración y barbarie: la construcción social y política del inmigrante como amenaza. Papers: revista de sociología,
66, $59-75$. doi: https://doi.org/10.5565/rev/papers/v66n0.1621

Sebastiani, L. (2015). Desenredando las políticas públicas de la Unión Europea. El conjunto "Principios Básicos Comunes”/“Fondo Europeo para la Integración” como una tecnología de gobierno. Política y Sociedad, 52(1), 205-232. doi: http://doi.org/209/rev_POSO.2015.v1.n52.457 $\underline{89}$

Simó, N., Pamies, J., Collet-Sabé, J. \& Tort, A. (2014). La acogida educativa en los centros escolares en Cataluña: más allá de los recursos específicos para el alumnado de nueva incorporación. Revista Complutense de Educación, 25(1), 177-194. doi: http://doi.org/10.5209/rev_RCED.2014.v25.nl. 41410

Siqués, C. \& Vila, I. (2014). Estrategias discursivas y aprendizaje de la lengua escolar: un estudio etnográfico en un aula de alta diversidad étnica y lingüística. Cultura y Educación: Revista de teoría, investigación y práctica, 26(2), 349-376. doi: https://doi.org/10.1080/11356405.2014.935112

Soriano, E. \& González, A. (2010). El poder educativo de las asociaciones de inmigrantes en las escuelas multiculturales. RELIEVE, 16(1), 1-20. doi: http://doi.org/10.7203/relieve.16.1.4150

Soriano, E. \& Peñalva, A. (2011). Presente y futuro de la educación intercultural en la formación inicial de los profesionales de la educación. Aula abierta, 39(1), 117-130. Consultado en http://redined.mecd.gob.es/xmlui/bitstream/han dle/11162/5202/01720113011761.pdf?sequenc $\underline{\mathrm{e}=1}$

Travé, G. (2013). Un estudio sobre las representaciones del profesorado de Educación Primaria acerca de la enseñanza bilingüe. Revista de Educación, 361, 379-402. doi: http://doi.org/10.4438/1988-592X-RE-2011361-149

Tubino, F. (2004). Del interculturalismo funcional al interculturalismo crítico. En M. 
Samaniego \& G. Garbarini (Eds.), Rostros y fronteras de la identidad (pp.1-9). Santiago de Chile: Universidad Católica de Temuco. Consultado en http://red.pucp.edu.pe/wpcontent/uploads/biblioteca/inter_funcional.pdf

Tubino, F. (2011). El nivel epistémico de los conflictos interculturales. Revista Electrónica Construyendo Nuestra Interculturalidad, 7 (67), 1-14. Consultado en http://interculturalidad.org/numero7/index.php/ es/ediciones-anteriores

Zapata-Barrero, R. (2001). Fundamentalismo estatal de la UE en torno a la inmigración. Revista CIDOB d'afers internacionals, 53, 149-176. Consultado en http://www.cidob.org/content/download/58485 /1519090/.../1/.../53zapata.pdf

Vertovec, Steven (2007). "Super-diversity and itsimplications". Ethnic and Racial Studies, 30(6), 1024-1054. doi: https://doi.org/10.1080/01419870701599465

Viaña, J. (2010). Fundamentos para una interculturalidad crítica. En L. Tapia, C. Walsh \& J. Viaña (Eds.), Construyendo interculturalidad crítica (pp. 97-133). La Paz:
Instituto Internacional de Integración del Convenio Andrés Bello.

Vila, I. (2000). Enseñar a convivir, enseñar a comunicarse. Revista Textos de Didáctica de la Lengua y de la Literatura, 23, 23-30.

Wallman, S. (2003). The Diversity of Diversity: Implications of the Form and Process of Localised Urban Systems. Nota di Lavoro, Social Sciencie Research Network. Electronic Paper Collections, 76, 1-22. Consultado en http://www.feem.it/userfiles/attach/Publication /NDL2003/NDL2003-076.pdf

Walsh, C. (2008). Interculturalidad crítica, pedagogía decolonial. En W. Villa \& A. Grueso (Comp.), Diversidad, interculturalidad y construcción de cuidad (s/p). Bogotá: Universidad Pedagógica Nacional y Alcaldía Mayor.

Walsh, C. (2012). Interculturalidad y (de)colonialidad: Perspectivas críticas. Visao Global, 15 (1-2), 61-74. Consultado en http://editora.unoesc.edu.br/index.php/visaoglo bal/article/viewFile/3412/1511

\section{Notas}

${ }^{[1]}$ Los principales instrumentos normativos que dan cobertura a la "Educación Intercultural" en el contexto estudiado son la Ley 9/1999, de Solidaridad en la Educación, el Decreto 167/2003, por el que se establece la ordenación educativa a los alumnos y alumnas con necesidades educativas especiales asociados a condiciones sociales desfavorecidas, el Plan Andaluz de Educación a Inmigrantes (2001), el Plan de Cultura de Paz y no Violencia (2002), el Plan Andaluz de fomento del Plurilingüismo (2005) y la Orden de 15 de enero de 2007, por la que se regulan las medidas y actuaciones a desarrollar para la atención del alumnado inmigrante y, especialmente, las Aulas Temporales de Adaptación Lingüística (2007). Todos ellos se han ido recogiendo paulatinamente en el Plan Integral para la Inmigración en Andalucía en sus distintas renovaciones (I para los años 20012004, II para 2006-2009 y III para 2014-2016). De igual manera se ha de hacer mención a las distintas políticas desarrolladas hasta el momento en materia de integración de la población inmigrante a nivel estatal (García \& Olmos-Alcaraz, 2010; Contini, Olmos-Alcaraz \& Contini, 2016) y las reducidas menciones que hacen las distintas leyes educativas estatales que han funcionado hasta la fecha (ver Rodríguez Navarro et al., 2011).

[2] Tubino (2004), desde el contexto latinoamericano, define interculturalismo funcional o neo-liberal como un modelo que defiende el diálogo y el reconocimiento de la diversidad sin cuestionar a fondo la situación de pobreza y desigualdad crónica en la que se encuentran los grupos culturales subalternos. Es decir, se trata de un modelo que no cuestiona el sistema y por lo tanto permite su reproducción siendo "funcional" al Estado nación y al capitalismo. Pone como ejemplo el multiculturalismo anglosajón, y nos habla de discriminación positiva y educación compensatoria como "programas paliativos a los problemas, [que] no generan ciudadanía, [y] que promueven la 
equidad pero desde arriba” (Tubino, 2004, p. 6), por lo que son, en definitiva paternalistas. Puede verse una reflexión en este sentido para el caso español en Olmos-Alcaraz \& Contini (2016).

[3] Los proyectos son "Éxitos y fracasos escolares. Trayectorias socioculturales de inmigrantes extranjeros y escolares autóctonos en el sistema educativo andaluza” (Ministerio de Ciencia e Innovación, Plan Nacional de I+D+i, 2010-2013), "Multiculturalidad e integración de la población inmigrante extranjera en las escuelas andaluzas" (Junta de Andalucía, Proyectos de Excelencia, 20072010) y "Evaluación de las actuaciones de atención educativa del alumnado inmigrante en Andalucía, Junta de Andalucía, 2004-2006), todos ellos dirigidos por el profesor Francisco Javier García Castaño. Todo ello actualmente está teniendo continuidad a través del Proyecto de Investigación "Construyendo diferencias en la escuela. Estudios de las trayectorias de las ATAL en Andalucía, de su profesorado y de su alumnado" (Ministerio de Ciencia e Innovación, Plan Nacional de I+D+i, 2014-2016).

${ }^{[4]}$ En un primer momento se seleccionaron los 10 centros educativos de Málaga, Almería y Granada con mayor porcentaje de alumnado extranjero, y tras una primera toda de contacto con los mismos se eligieron para realizar los cuestionarios los que presentaban mayor accesibilidad y predisposición para colaborar en la investigación.

${ }^{[5]}$ Una explotación detallada del cuestionario utilizado puede verse en Olmos-Alcaraz (2010b).

${ }^{[6]}$ Las encuestas fueron aplicadas a los estudiantes por las investigadoras Ouafaa Bouachra Outmani, María Rubio Gómez y Mónica Ortiz Cobo.

${ }^{[7]}$ Dado que los objetivos de este artículo con respecto a los datos obtenidos a partir del cuestionario se circunscriben a visibilizar la diversidad lingüística del alumnado entrevistado (objetivos de carácter descriptivo) las pruebas estadísticas realizadas responden a ello, limitándose a ofrecer datos porcentuales a partir de tablas de frecuencias y de contingencia.

${ }^{[8]}$ Para ello se confeccionó una plantilla de categorías temática que facilitó el análisis de los discursos. Análisis e interpretación de la información se produjeron de forma ligada y en ocasiones simultánea, dadas las características -etnográficas- del trabajo.

${ }^{[9]}$ Es preciso cuestionar y de-construir la forma misma de referirnos a un proceso (inmigrar) como si fuese un estado perpetuo (ser “inmigrante”). ¿Hasta cuando se es pues “inmigrante”? Mientras dura el proceso de migrar, el desplazamiento... habríamos de pensar. Sin embargo la realidad es otra. La categoría demográfica "inmigrante" en el contexto estudiado ha sufrido una transformación que la hace parecer una categoría socio-cultural cargada de significados negativos y que se mantiene en el tiempo de manera indefina para ciertos colectivos, hasta llegar a hablarse de "segundas generaciones" de inmigrantes para referir a personas que nunca se desplazaron.

${ }^{[10]}$ La nacionalidad ecuatoriana y colombiana se encuentran entre las más numerosas en el momento actual en España, suponiendo un 5\% (240.735) y un 4\% (194.812) respectivamente de la población extranjera total (Instituto Nacional de Estadística).

${ }^{\text {[11] }}$ Para el último curso del que disponemos de datos (2013-14) se cuentan con 250 profesores/as de ATAL en toda Andalucía. Se ha mantenido el número de cursos anteriores, después de registrar una paralización del incremento de los mismos durante los últimos años. No obstante, no se han disminuido los efectivos, a pesar de que la financiación estatal para ello ha desaparecido (Junta de Andalucía, 2013).

${ }^{[12]}$ Datos del Ministerio de Educación y Ciencia, para el curso 2011-12.

[13] Es interesante en este sentido los resultados obtenidos por en una investigación sobre las percepciones del profesorado acerca de este plan lingüístico: el 81\% de los profesores se mostraban contrarios a la idea de incluir "otras” lenguas en dicho programa (Travé, 2013).

${ }^{[14]}$ En este sentido se ha de mencionar que existen en la comunidad diversos programas dentro de lo que se conoce como ELCO (Enseñanza de la Lengua y Cultura de Origen) a través de los cuales se ofrecen clases de marroquí y de rumano. Estas clases se imparten en horario extra-escolar, contando para ello en el curso 2013-14 con 20 profesores de nacionalidad marroquí y 6 de nacionalidad rumana 
para toda la comunidad (Junta de Andalucía, 2013). En cursos anteriores estaban incluidos en los programas ELCO la enseñanza del chino y del portugués, pero recientemente dichas lenguas tienen sus propios programas, fuera de ELCO, aunque siguen siendo impartidos en horario extra-curricular. Todos los programas señalados tienen una cobertura muy modesta del territorio.

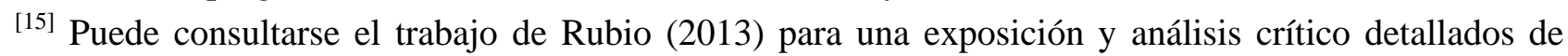
dichos programas existentes en Andalucía para los niveles de secundaria. Los mismos pueden dividirse, a grandes rasgos, en: 1. Programas de carácter organizativo; Programas de adaptación curricular; y 3. Programas de atención específica a la diversidad de "los/as inmigrantes” en la escuela.

${ }^{[16]}$ Recientemente, dentro del VIII Congreso Internacional sobre Migraciones, se organizó un simposio sobre aulas especiales para la acogida de inmigrantes. En el mismo, y en marco de la investigación citada más arriba denominada "Construyendo diferencias en la escuela. Estudios de las trayectorias de las ATAL en Andalucía, de su profesorado y de su alumnado" (Ministerio de Ciencia e Innovación, Plan Nacional de I+D+i, 2014-2016), se presentaron varios trabajos donde se reflexionaba y se ponía en valor el trabajo realizado por estos profesionales. Algunas de las presentaciones fueron realizadas incluso por el propio profesorado y por responsables técnicos de la Administración Educativa, lo cual no suele ser habitual en estos ámbitos académicos y puede ser muestra del inicio de un diálogo entre distintos agentes implicados. Pueden consultarse las actas de dicho congreso para conocer estos trabajos.

Olmos-Alcaraz, Antonia (antonia@ugr.es).

Profesora Ayudante Doctora en el Departamento de Antropología Social de la Universidad de Granada. Investigadora del Instituto de Migraciones, y dentro de este, perteneciente al grupo de investigación "Laboratorio de Estudios Interculturales" (SEJ-123). Sus líneas de investigación incluyen Políticas Migratorias, Educación Intercultural, Alteridad/Identidad. Dirección de contacto: Facultad de Ciencias de la Educación, Campus de Cartuja, s/n, 18071, Granada.

RELIEVE

\section{Revista ELectrónica de Investigación y EValuación Educativa E-Journal of Educational Research, Assessment and Evaluation}

[ISSN: 1134-4032]

(C) Copyright, RELIEVE. Reproduction and distribution of this articles it is authorized if the content is no modified and their origin is indicated (RELIEVE Journal, volume, number and electronic address of the document).

(C) Copyright, RELIEVE. Se autoriza la reproducción y distribución de este artículo siempre que no se modifique el contenido y se indique su origen (RELIEVE, volumen, número y dirección electrónica del documento). 